\title{
Deployment Analysis of Carrier Grade Urban Wireless Mesh Networks
}

\author{
J. I. E. Lee ${ }^{1}$, D. Chieng ${ }^{1}$, M. Gruber ${ }^{2}$ \\ ${ }^{I}$ Malaysian Research Centre, BT Innovate and Design, British Telecommunications Plc \\ Kuala Lumpur, Malaysia \\ \{itee.lee, david.chieng\}@bt.com \\ ${ }^{2}$ Alcatel-Lucent Bell Labs \\ Stuttgart, Germany \\ markus.gruberdalcatel-lucent.de
}

\begin{abstract}
An optimized and cost-effective wireless mesh network (WMN) deployment for ubiquitous broadband wireless access is known to be highly dependent on a number of key factors such as backhaul solution, number of radio interfaces per node, type of radio technology, network topology/cluster, etc. The choice of network topology in turn affects the gateway-node ratio, and also the placement of gateways $(G W)$ s and access points (AP)s. In this paper, we provide insights on the best possible upper-bound performance and inter-relationship between these key design factors under different operating conditions with respect to different target data rates per user. Next, we incorporate deployment cost analysis into the corresponding design option studies in order to provide more accurate justification on the feasibility of adopting different clustering techniques. The results from our analysis offer a general guideline or reference for network operators who intend to deploy a uniform blanket coverage using WMN especially in the urban environment.
\end{abstract}

Keywords — wireless mesh networks; wireless backhaul systems; infrastructure mesh topologies; clustering; CAPEX/OPEX

\section{INTRODUCTION}

In the recent decade, the ever-increasing commercial demand to pursue unlimited high-speed and ubiquitous broadband wireless access has spurred numerous extensive research efforts on mesh networking and rapid adoption of wireless mesh networks (WMNs) utilizing various wide-area broadband wireless access technologies such as WirelessFidelity (Wi-Fi) and Worldwide Interoperability for Microwave Access (WiMAX). The realization of wide-area broadband wireless access relies upon a large number of base stations (BS)s which are uniformly scattered over a specific geographical area to provide the required wireless coverage, due to the inverse relationship between the data rate and BS range as well as the limitations on range imposed by the corresponding BS transmit power [1]. Another important factor that will validate the feasibility of such an approach is the availability of backhaul connectivity to a large number of BSs within the desired coverage area. Consequently, wireless backhaul system incorporating mesh or multi-hop technology is poised to become a key player in providing the necessary backhaul solution to various broadband wireless access networks.
In general, mesh networking refers to the routing of information from source to destination by means of multiple wireless links, whereby this concept has been studied extensively in the context of wireless ad hoc networks. Research studies revealed the potential advantages of the multi-hop technology over traditional single-hop networking, such as spatial reuse for increased capacity, coverage enhancement [2-[4], load balancing through route diversity [5] and user cooperation diversity, which in turn reflected the need to integrate mesh networking into the wireless backhaul system to establish backhaul communication. Nevertheless, optimization of the wireless backhaul mesh network capacity and scalability requires more feasible and effective network topology planning methodologies and techniques, in order to enable strategic positioning and placement of GWs, BSs/APs and relay stations (RS)s, due to the fact that traffic increases with both the number of nodes and distance over which each node wishes to communicate (i.e., due to packet forwarding).

Our present research effort aims to derive a proper mesh network planning strategy in the context of CARMEN. Indepth simulation studies have been carried out to evaluate the best possible upper-bound performance and the interrelationship of various design options such as target data rate per user, infrastructure mesh topology formation/clustering technique, node density and gateway-node ratio under different operating conditions and cost factors. Correspondingly, these findings could represent a general rule-of-thumb for network operators to enable a cost-effective greenfield deployment of WMNs with uniform blanket coverage in urban communities

The remainder of this article is structured as follows: Section II describes the system model, in which the network architecture and technical model taken into account in our analysis are described in detail here. In addition, various assumptions made in the corresponding studies are highlighted and explained accordingly in this section. Next, the different infrastructure mesh topologies considered in our studies are introduced in Section III. Simulation results are presented and discussed in Section IV before we conclude this paper in Section V. 


\section{SYSTEM MODEL}

\section{A. Network Architecture and Assumptions}

In the present simulation studies, we consider an outdoor two-tier infrastructure/backbone WMN (Fig. 1) [6, 7], which comprises a backhaul tier for providing wired network connectivity to the infrastructure mesh nodes and an access tier for enabling wireless communication between mesh nodes and client devices. Within the backhaul tier, a fraction of these mesh nodes with gateway functionality feature a wired connection to the Internet, thus representing sources/sinks in the WMN. Correspondingly, data traffic is forwarded from source to destination via multihop paths formed by mesh nodes.

In our effort to derive useful high-level insights pertaining the best possible upper bound capacity performance, while conforming to feasible and cost-effective design options for typical greenfield deployment of WMNs with uniform blanket coverage, the following assumptions have been made:

1) Fair backhaul and access bandwidth: It is assumed that the total backhaul capacity is equally distributed among the infrastructure mesh nodes, thereby implying a fair share of access to the wireless medium across some fixed timeslots between the user domains and mesh nodes attached to the same backhaul point. Consequently, the access bandwidth is evenly disseminated among the user domains to ensure a uniform blanket deployment of wireless broadband services, whereby mesh nodes with greater hops away from the GW enjoy the same bandwidth share as compared to those nodes positioned nearer to the GW. Various techniques such as call admission control and fair scheduling [8] have been proposed to make this possible.

2) Negligible RF interferences: We assume that interference arising from co-channel, adjacent channel, foreign devices, etc., are negligible in situations where there are sufficient orthogonal channels, and/or the network operates under licensed spectrum [9]. In addition, the effects of interferences can be eliminated through the application of effective frequency planning approaches and introduction of smart antenna systems (e.g., beam forming) between mesh

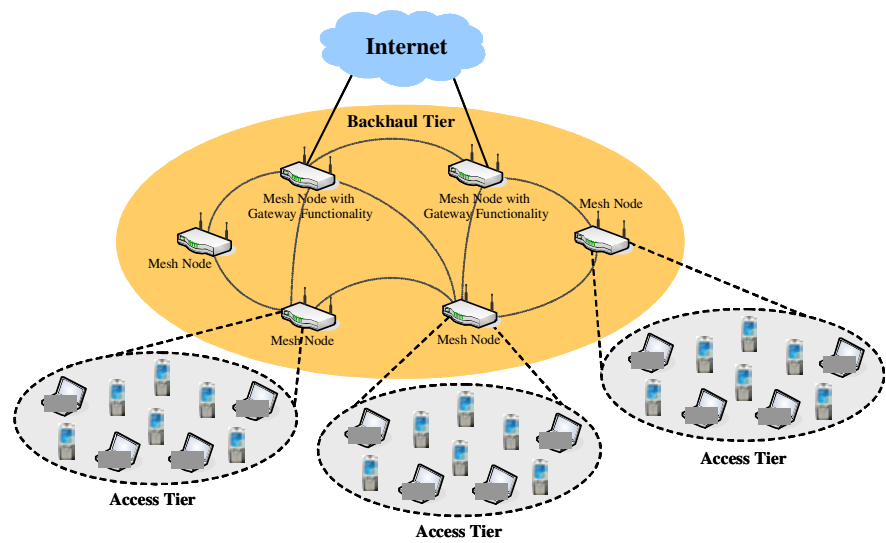

Fig. 1 A two-tier infrastructure/backbone wireless mesh network comprising a backhaul tier for providing wired network connectivity to the infrastructure mesh nodes and an access tier for enabling wireless communication between mesh nodes and client devices. nodes. Albeit their practical limitations at the present stage, such assumptions present fundamental requirements for estimating the upper-bound performance limits of wireless mesh networking systems, which are critical considerations for network operators to rationalize on the optimized capacity performance of wireless mesh systems and the viability of this technology under licensed spectrum environment [9].

3) Ideal routing mechanism: When network dynamic is at statistical equilibrium, a mesh network is behaving like a multihop network based on the assumption that the network is supported by an effective routing scheme which provides uniform or fair capacity distribution (i.e., load balancing) across all the nodes within a cluster.

4) One user domain per infrastructure mesh node: We assume that each infrastructure mesh node is assigned with one access link and serves only one user domain, thus resulting in the following implications: (1) all mesh nodes within the backhaul tier have packet forwarding capabilities but are not merely relay nodes; and (2) all end users within a particular user domain are required to share the same access point.

\section{5) Multi-radio system:}

In a multiple radio system, each infrastructure mesh node is equipped with $T_{R}=N+1$ radio interfaces, in order to create independent backhaul links with its corresponding $N$ neighbouring nodes and to provide wireless access to its own user domain, whereby this multi-radio node can concurrently transmit (or receive) traffic to each of its neighbours as well as to end users subscribing within its domain.

\section{B. Channel Model}

The physical layer model used to determine the measurement-parameterized link behaviour is a well-known pathloss model [10], which relates link distance to an expected signal strength on that link as given by the relation

$$
P L(d B)=-147.6+20 \log \left(f_{R F}\right)+10 \alpha \log (d)
$$

where $d$ denotes the distance between the transmitting and receiving nodes (in $\mathrm{m}$ ), $f_{R F}$ represents the signal frequency (in $\mathrm{Hz}$ ) and $\alpha$ resembles the path loss exponent.

Correspondingly, the theoretical baseband received signal power to noise ratio $S N R$ (in $\mathrm{dB}$ ) can be determined from the receiver sensitivity $R_{\text {sen }}$ (in $\mathrm{dBm}$ ), which is defined as the received signal power needed to operate at a given bit-errorrate (BER) and described by the following expression [11]:

$$
\begin{aligned}
R_{\text {sen }}= & \frac{k T_{A}}{2} \cdot S_{\text {wireless }} \cdot N_{\text {figure }} \cdot I_{\text {implementation }} \cdot S N R \\
= & -177+10 \log \left(S_{\text {wireless }}\right)+10 \log \left(N_{\text {figure }}\right) \\
& +10 \log \left(I_{\text {implementation }}\right)+10 \log (S N R)
\end{aligned}
$$

in which its value is further derived from the link budget calculation as follow:

$$
P L=E I R P-R_{\text {sen }}+G_{r x}-M_{\text {total }}
$$


where $k$ is Boltzmann constant, $T_{A}$ denotes the absolute temperature, $S_{\text {wireless }}$ is the wireless link symbol rate, $N_{\text {figure }}$ represents the noise figure, $I_{\text {implementation }}$ is the degradation caused by implementation limitations, EIRP resembles the effective isotropic radiated power, $G_{r x}$ is the receiver antenna gain and $M_{\text {total }}$ is the total margin.

Assuming the adoption of WiMAX air interface with full usage of subcarriers (FUSC), the parameters employed in the link budget computation are defined accordingly in Table I. Based on the calculated SNR, the optimized modulation and coding scheme (MCS) that the channel can support at BER of $10^{-6}$ with convolutional turbo code (CTC) in additive white Gaussian noise (AWGN) channel [11] is then determined from the required SNR thresholds for the respective MCS as tabulated in Table II, which also presents the corresponding ideal and effective data rates as well as receiver sensitivity.

\section{INFRASTRUCTURE MESH TOPOLOGIES}

Performance and cost-effectiveness of WMNs deployment with uniform blanket coverage relies upon various design parameters such as density of nodes (including gateways), total backhaul capacity and number of radio interfaces, which are later translated into equivalent deployment cost. Hence, an optimized and low-cost topology design motivates the current effort to evaluate the viability of numerous infrastructure mesh topologies (or cluster design) ranging from one hop to four hops.

In our analysis, it is assumed that the coverage area $A_{\text {node }}$ served by each BS is a hexagonal cell which can be determined from the equation:

$$
A_{\text {node }}=\frac{\sqrt{3}}{2} D_{\text {mesh }}^{2}
$$

in which the distance between two mesh nodes $D_{\text {mesh }}$ is given by :

$$
D_{m e s h}=2 R \cos 30^{\circ}=R \sqrt{3}
$$

where $R$ denotes the cell radius. $M_{\text {total }}$ BSs form a cluster in such a way that they tessellate the plane with the following cluster area $A_{\text {cluster }}$ :

$$
A_{\text {cluster }}=M_{\text {total }} \times \frac{\sqrt{3}}{2} D_{m e s h}^{2} .
$$

TABLE I. PRoPerties OF THE LiNK BUdGeT

\begin{tabular}{lr}
\hline \multicolumn{1}{c}{ Parameter } & Value \\
\hline RF frequency & $2.5 \mathrm{GHz}$ \\
Channel bandwidth & $20 \mathrm{MHz}$ \\
Transmit EIRP power & $63 \mathrm{dBm}$ \\
Receiver antenna gain & $17 \mathrm{~dB}$ \\
Noise Figure & $8 \mathrm{~dB}$ \\
Thermal noise power density & $-174 \mathrm{dBm} / \mathrm{Hz}$ \\
Implementation loss & $5 \mathrm{~dB}$ \\
Link layer efficiency & 1 \\
DL:UL & $3: 1$ \\
Total margins & \\
Interference & $3 \mathrm{~dB}$ \\
Fading & $3 \mathrm{~dB}$ \\
Total number of tones & 2048 \\
Number of data tones & 1536 \\
Number of pilot tones & 166 \\
Number of guard tones & 346 \\
Sampling factor & $28 / 25$ \\
Wireless symbol rate (consider data and & $18.64 \mathrm{MS} / \mathrm{s}$ \\
pilot only) &
\end{tabular}

In general, the number of cells that can form a regular $M_{\text {total }}$-sized cluster pattern which comprises one GW and $\left(M_{\text {total }}-1\right)$ BSs is given by the formula $M_{\text {total }}=m^{2}+n^{2}+m n$, where $m$ and $n$ are integers, thereby giving $M_{\text {total }}=3,4,7$, $9,12,13,16,19,21$, etc [12]. Since the topology design considered in our analysis assumes one $\mathrm{GW}$ positioned at the centre of the cluster is surrounded by numerous BSs that form a symmetrical-like multihop pattern, the cluster sizes selected are 4, 7, 13, 19 and 37 with corresponding GW-to-AP ratio of $1: 3,1: 6,1: 12,1: 18$ and 1:36, respectively, in which the design options under study can be configured with limited 1 hop (i.e., no forwarding) to maximum 4 hops. Fig. 2 illustrates the layout of a 1:6 topology design where 6 APs are connected wirelessly to $1 \mathrm{GW}$ with wired backhaul at the centre of the cluster, which can be configured with minimum 1 hop (6 branches) and maximum 3 hops ( 2 branches).

The same performance can be achieved without the need to exhibit the exact symmetry as shown in Fig. 2 since the link capacity between two nodes is predominantly governed by the signal quality (e.g. SNR). This hence implies in the actual deployment, some degree of perturbation in terms of the location of the nodes is allowed without affecting the results obtained in the following sections.

Refer to Table III for a detailed breakdown of different infrastructure mesh topology design options considered in our studies.

TABLE II. THE REQUIRED SNR THRESHOLDS FOR THE RESPECTIVE MODULATION AND CODING SCHEME AND THEIR CORRESPONDING IDEAL AND EFFECTIVE DATA RATES AND RECEIVER SENSITIVITY

\begin{tabular}{ccccc}
\hline \hline $\begin{array}{c}\text { Modulation and } \\
\text { Coding Scheme }\end{array}$ & $\begin{array}{c}\text { SNR Threshold } \\
(\mathbf{d B})\end{array}$ & $\begin{array}{c}\text { Ideal Data-Rate } \\
(\mathbf{M b p s})\end{array}$ & $\begin{array}{c}\text { Effective Data } \\
\text { Rate (Mbps) }\end{array}$ & $\begin{array}{c}\text { Receiver Sensitivity, } \\
\boldsymbol{R}_{\text {sen }}(\mathbf{d B m})\end{array}$ \\
\hline 1/2 QPSK & 2.9 & 15.00 & 11.25 & -88.37 \\
3/4 QPSK & 6.3 & 22.50 & 16.88 & -84.97 \\
1/2 16-QAM & 8.6 & 30.00 & 22.50 & -82.67 \\
3/4 16-QAM & 12.7 & 45.00 & 33.75 & -78.57 \\
1/2 64-QAM & 13.8 & 45.00 & 33.75 & -77.47 \\
2/3 64-QAM & 16.9 & 60.00 & 45.00 & -74.37 \\
3/4 64-QAM & 18.0 & 67.50 & 50.63 & -73.27 \\
5/6 64-QAM & 19.9 & 75.00 & 56.25 & -71.37 \\
\hline
\end{tabular}






(a)



(b)

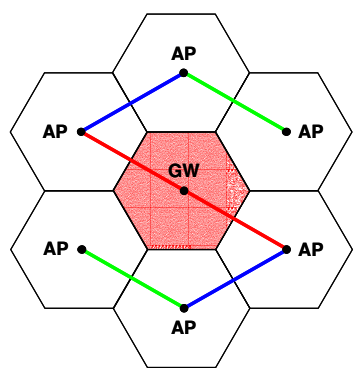

(c)

Fig. 2 The layout of a 1:6 topology design where 6 APs are connected wirelessly to $1 \mathrm{GW}$ with wired backhaul at the center of the cluster, which can be configured with (a) minimum 1 hop (6 branches); (b) 2 hops ( 3 branches); and maximum 3 hops ( 2 branches).

\section{SIMULATION RESULTS AND DISCUSSION}

Fig. 3 describes the linear relationship between average data rate per user and node density and highlights the impact of multihop technology on the network performance, in which these observations present useful guidelines for network operators to forecast the size of the network required, in order to provide uniform blanket coverage while achieving the

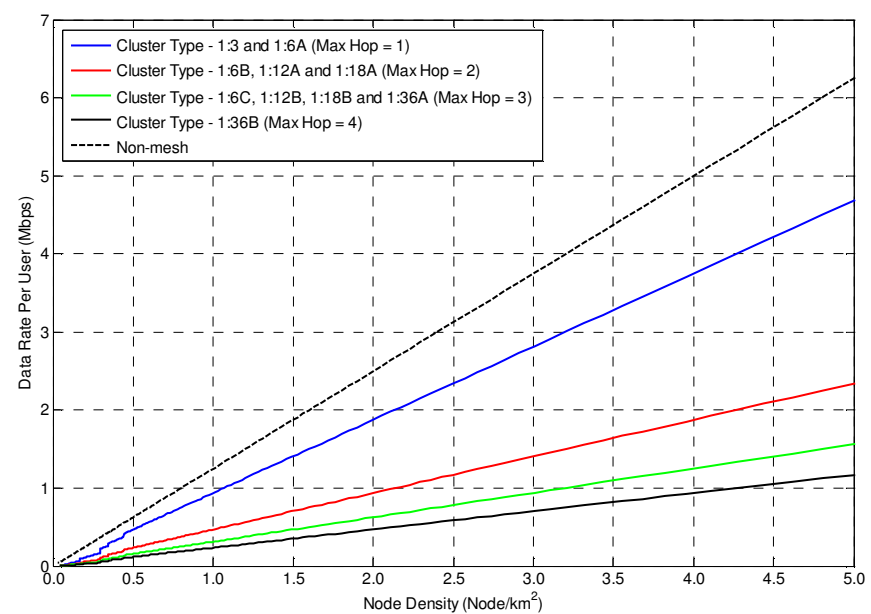

Fig. 3 The relationship between average data rate per user and node density for various topology design options, which highlighted the impact of multihop technology on network performance.

average data rate per user requirement. Our findings reveal that the broadband wireless access service enjoyed by end users degrades with greater number of hops between the source and destination nodes (i.e., number of APs sharing the same multihop branch), where significant performance gap is noted between 1-hop and 2-hop configurations. As a result, mesh topology design with greater maximum hop count per branch would require higher node density to deploy a WMN operating at the desired data rate per user. For instance, the 1:36B cluster design requires a significantly higher node density of 4.27 node $/ \mathrm{km}^{2}$ as compared to design options with 1-hop configuration (i.e., $1: 3$ and 1:6A) with the lowest density of 1.07 node $/ \mathrm{km}^{2}$.

Fig. 4 illustrates the variation of data rate per user with

TABLE III. BREAKDOWN OF DIFFERENT INFRASTRUCTURE MESH TOPOLOGY DESIGN OPTIONS

\begin{tabular}{|c|c|c|c|c|c|c|c|c|c|c|}
\hline \multirow{2}{*}{ Parameter } & \multicolumn{10}{|c|}{ Cluster Type } \\
\hline & $1: 3$ & $1: 6 \mathrm{~A}$ & $1: 6 B$ & $1: 6 \mathrm{C}$ & $1: 12 \mathrm{~A}$ & $1: 12 B$ & $1: 18 A$ & $1: 18 B$ & $1: 36 A$ & $1: 36 B$ \\
\hline \multicolumn{11}{|l|}{ Cluster Metrics: } \\
\hline Total number of GWs & 1 & 1 & 1 & 1 & 1 & 1 & 1 & 1 & 1 & 1 \\
\hline Total number of APs & 3 & 6 & 6 & 6 & 12 & 12 & 18 & 18 & 36 & 36 \\
\hline Total number of nodes & 4 & 7 & 7 & 7 & 13 & 13 & 19 & 19 & 37 & 37 \\
\hline GW:AP ratio & $1 / 3$ & $1 / 6$ & $1 / 6$ & $1 / 6$ & $1 / 12$ & $1 / 12$ & $1 / 18$ & $1 / 18$ & $1 / 36$ & $1 / 36$ \\
\hline Total nodes at Hop 1 & 1 & 6 & 3 & 2 & 6 & 3 & 6 & 3 & 6 & 3 \\
\hline Total nodes at Hop 2 & 0 & 0 & 3 & 2 & 6 & 3 & 12 & 6 & 12 & 6 \\
\hline Total nodes at Hop 3 & 0 & 0 & 0 & 2 & 0 & 6 & 0 & 9 & 18 & 11 \\
\hline Total nodes at Hop 4 & 0 & 0 & 0 & 0 & 0 & 0 & 0 & 0 & 0 & 16 \\
\hline Total radios at GW & 4 & 7 & 4 & 3 & 7 & 4 & 7 & 4 & 7 & 4 \\
\hline Total radios at Hop 1 & 2 & 2 & 3 & 3 & 3 & 3 & 4 & 4 & 4 & 4 \\
\hline Total radios at Hop 2 & 0 & 0 & 2 & 3 & 2 & 4 & 2 & 4 & 4 & 4 \\
\hline Total radios at Hop 3 & 0 & 0 & 0 & 2 & 0 & 2 & 0 & 2 & 2 & 4 \\
\hline Total radios at Hop 4 & 0 & 0 & 0 & 0 & 0 & 0 & 0 & 0 & 0 & 2 \\
\hline Total number of branches & 3 & 6 & 3 & 2 & 6 & 3 & 6 & 3 & 6 & 3 \\
\hline Total nodes per branch & 1 & 1 & 2 & 3 & 2 & 3 & 2 & 3 & 3 & 4 \\
\hline \multicolumn{11}{|l|}{ Deployment Metrics*: } \\
\hline Total number of GWs & 2669 & 1525 & 3095 & 4582 & 1667 & 2467 & 1140 & 1688 & 867 & 1154 \\
\hline Total number of nodes & 10676 & 10676 & 21668 & 32075 & 21668 & 32075 & 21668 & 32075 & 32075 & 42703 \\
\hline Node density (node $/ \mathrm{km}^{2}$ ) & 1.07 & 1.07 & 2.17 & 3.21 & 2.17 & 3.21 & 2.17 & 3.21 & 3.21 & 4.27 \\
\hline
\end{tabular}


increasing active subscribing population for different clustering techniques with maximum hop count per branch ranging from 1 to 4 , assuming that the subscribers access the network simultaneously, thereby quantifying the interrelationship of the following design options - target data rate, cluster type and maximum percentage (or number) of active subscribers that can be supported. It should be noted that the total number of active subscribers represents the worst case for a given subscriber base. Hence, this result could be employed by network operators as a general rule-of-thumb for selecting the most optimized mesh topology design, which can provide coverage to a targeted percentage of population with the desired data rate requirement. For example, the 1:36B topology design can support a maximum active subscribing population of $10 \%$ for a data rate requirement of $1 \mathrm{Mbps}$, but the cluster type is expected to evolve to design options with lower maximum hop count per branch as the number of active subscribers increases, in order to maintain the same data rate requirement.

The previous analysis which outlines the inter-relationship of various design options such as data rate per user requirement, node density, maximum active subscribing population and clustering technique will be meaningful for network operators with the incorporation of substantial cost analysis. The corresponding cost analysis assumes a data rate per user requirement of $1 \mathrm{Mbps}$, and defines total deployment cost as the sum of CAPEX and OPEX (for 1 year) which is calculated based on the price listings extracted from [13-[15]. Fig. 5 presents the relationship between total deployment cost and node density for different mesh topology options, and highlights the breakdown of various cost components involved, which in turn reveals a greater contribution of CAPEX to the resulting cost due to a considerably larger node CAPEX. On the other hand, the OPEX has a smaller impact on the overall deployment cost due the least significant contribution of node OPEX, in which the relatively smaller cost component is justified by the absence of rental cost, since we assume that the site has been permanently acquired by the network operator. In addition, our observations indicate that

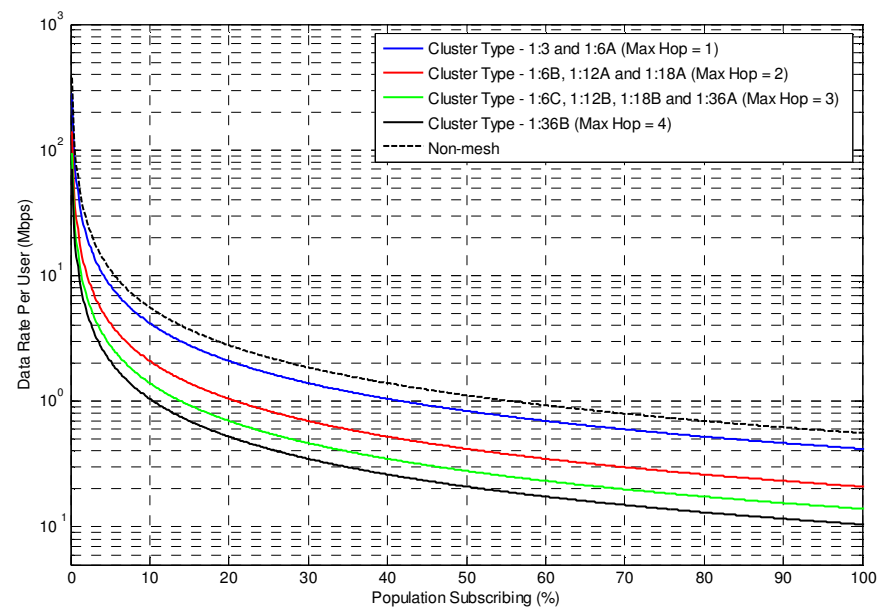

Fig. 4 Variation of data rate per user with increasing active subscribing population for different clustering techniques with maximum hop count per branch ranging from 1 to 4 .

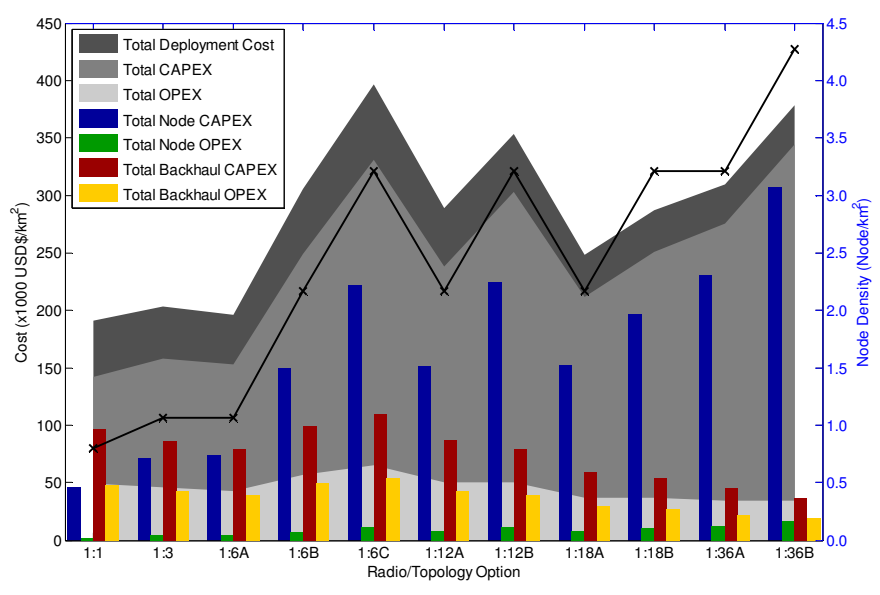

Fig. 5 The relationship between total deployment cost and node density for different mesh topology options, where total cost $=$ total CAPEX + total OPEX, total CAPEX = node CAPEX + backhaul CAPEX and total OPEX = node OPEX + backhaul OPEX.

the total deployment cost is very sensitive to the backhaul costs, especially for the non-mesh and cluster types with smaller number of hop count per branch (i.e., 1:3 and 1:6A) albeit exhibiting similar total cost. The corresponding findings from Fig. 5 justify that the 1:6C, 1:12B and 1:36B configurations are best to be avoided due to the significantly higher CAPEX and insignificant savings by comparison in terms of backhaul cost (CAPEX and OPEX). Nevertheless, part of the network may need to adopt such cluster options, particularly in situations when sites are limited due to legacy, geographical or structural constraints (buildings, etc), in which such conclusion is deduced under the assumption of 1 Mbps per user for the total urban population. Better conclusions can only be derived with more accurate cost figures and also to compare and contrast with different backhaul solutions and pricings.

Taking into consideration a uniform blanketed mesh deployment with node density of 1 node/km2, Fig. 6 shows that topology design options with larger maximum hop count per branch promote greater reductions in the overall deployment cost. The 1:36B topology design incurs the lowest cost when the number of subscribers is low at about $23 \%$ of the population in the area. As the subscriber base grows, the cluster types can be gradually migrated to $1: 18,1: 12$ or $1: 6$ respectively. The choice of which topology design to choose very much depending on additional installation cost which is not addressed here. Logically, it should be less expensive to migrate from $1: 36 \mathrm{~B}$ to $36 \mathrm{~A}$, compared to from $1: 36 \mathrm{~B}$ to $1: 18 \mathrm{~B}$ as the later not only needs to reconfigure the radio interfaces but also to add backhaul equipment. Similarly, better conclusions can only be derived with more accurate cost figures and also to compare and contrast with different backhaul solutions and pricings.

\section{CONCLUSIONS AND FUTURE WORKS}

In this paper, in-depth simulation studies have been carried out to evaluate the best possible upper-bound performance and the inter-relationship of various design options such as target 


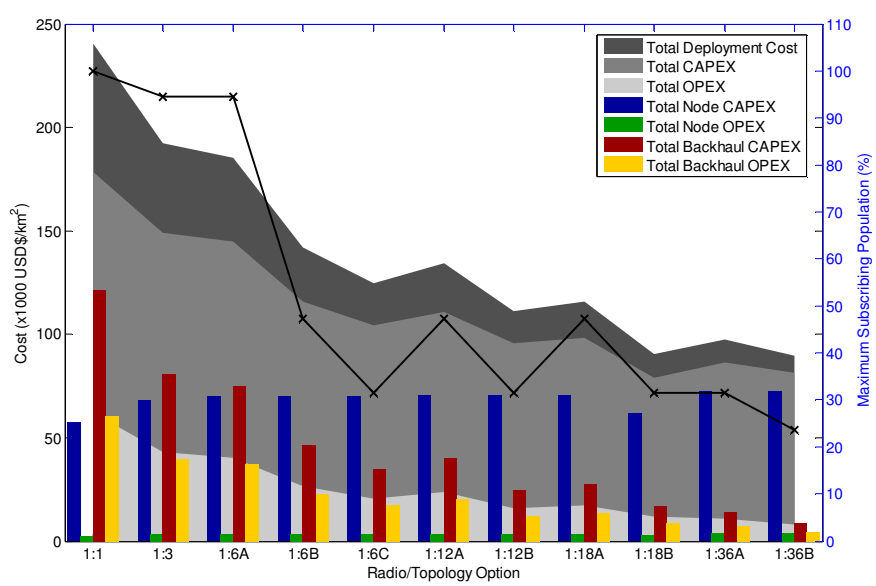

Fig. 6 TOPOLOGY design options with larger maximum hop count per branch support a relatively smaller percentage of subscribing population, but promote greater reductions in the overall deployment cost, assuming uniform blanketed mesh deployment with data rate per user requirement of $1 \mathrm{Mbps}$ and node density of 1 node $/ \mathrm{km} 2$.

data rate per user, infrastructure mesh topology formation/clustering technique, node density and gateway ratio under different operating conditions and cost factors. The results and findings from our analysis could be employed as a general reference or guideline for network operators who intend to deploy a uniform blanket coverage using WMN in urban environment while conforming to the desired user data rate requirements.

Our observations revealed that the average data rate per user degrades with greater maximum hop count per branch, thereby implying a higher node density to achieve the desired data rate requirement. In addition, the inter-relationship of various design options such as target data rate, cluster type and maximum percentage of active subscribing population could allow network operators to select the most optimized mesh topology design, which can provide coverage to a targeted percentage of population with the desired data rate requirement.

Furthermore, we have incorporated deployment cost analysis into the corresponding design option studies, in order to quantify the feasibility of adopting different cluster options. Our studies indicated that the total deployment cost is very sensitive to the backhaul costs, especially for the non-mesh and cluster types with smaller number of hop count per branch, and suggested that the $1: 6 \mathrm{C}, 1: 12 \mathrm{~B}$ and $1: 36 \mathrm{~B}$ configurations are best to be avoided due to the significantly higher CAPEX and insignificant savings by comparison in terms of total backhaul cost. Moreover, it is noted that the 1:36B topology design incurs the lowest cost when the number of subscribers is low at about $23 \%$ of the population in the area, and can be conveniently migrated to smaller sized cluster types such as $1: 18,1: 12$ or $1: 6$ as the subscriber base grows.

Nonetheless, there are many interesting design options that are worthwhile for further investigation, which includes the usage of advanced antenna systems such as MIMO and potential benefits of two-tier mesh architecture (i.e., using WMAN mesh such as WiMAX mesh to backhaul WiFi mesh clusters).

\section{ACKNOWLEDGMENT}

The research leading to these results has received funding from the EU Framework 7 Programme under grant agreement No. 214994. http://www.ict-carmen.eu/

The views and conclusions contained here are those of the authors and should not be interpreted as necessarily reflect those of BT, and also should not be interpreted as necessarily representing the official policies or endorsements, either expressed or implied, of the CARMEN project or the European Commission.

\section{REFERENCES}

[1] H. Viswanathan and S. Mukherjee, "Throughput-range tradeoff of wireless mesh backhaul networks”, IEEE J. Sel. Areas Commun., vol. 24, no. 3, pp. 593-602, Mar. 2006.

[2] S. Toumpis and A. J. Goldsmith, "Capacity regions for wireless ad hoc networks", IEEE Trans. Wireless Commun., vol. 2, no. 4, pp. 736-748, Jul. 2003.

[3] C. Jaeweon and Z. J. Haas, "On the throughput enhancement of the downstream channel in cellular radio networks through multihop relaying", IEEE J. Sel. Areas Commun., vol. 22, no. 7, pp. 1206-1219, Sep. 2004.

[4] H. Viswanathan and S. Mukherjee, "Performance of cellular networks with relays and centralized scheduling", IEEE Trans. Wireless Commun., vol. 4, no. 5, pp. 2318-2328, Sep. 2005.

[5] H. Wu, C. Qiao, S. De and O. Tonguz, "Integrated cellular and ad hoc relaying systems: iCAR", IEEE J. Sel. Areas Commun., vol. 19, no. 10, pp. 2105-2115, Oct. 2001.

[6] I. F. Akyildiz and X. Wang, "A survey on wireless mesh networks", IEEE Commun. Mag., vol. 43, no. 9, pp. S23-S30, Sept. 2005.

[7] J. Robinson and E. Knightly, "A performance study of deployment factors in wireless mesh networks, in Proc. INFOCOM, May 2007, pp. 2054-2062.

[8] N. Ben Salem, J. P. Hubaux, "A fair scheduling for wireless mesh networks", in Proc. IEEE Workshop on Wireless Mesh Networks (WiMesh), 2005.

[9] A. Ting and D. Chieng, "Design and capacity performance analysis of wireless mesh network", in Proc. International Conference on Mobile Technology, Applications and Systems (Mobility), Sep. 2008.

[10] T. S. Rappaport, Wireless Communications - Principles and Practice. Upper Saddle River, NJ: Prentice Hall, 2002.

[11] WiMAX FORUM. 2007. WiMAX Forum Mobile System Profile, Release 1.0 Approved Specification, revision 1.4.0.

[12] V. Gunasekaran and F. C. Harmantzis, "Affordable infrastructure for deploying WiMAX Systems: Mesh v. Non Mesh", in Proc. VTCSpring, May/June 2005, pp. 2979-2983.

[13] WiMAX: The Business Case for Fixed Wireless Access in Emerging Market, June 2005, WiMAX forum.

[14] BT Openreach Backhaul Extension Service (BES), Ethernet Products. http://www.openreach.co.uk/orpg/pricing/loadProductPriceDetails.do? data=kxWGSeZ9BRMKF3tctK4117\%2FuVhXjMR5hQz3DdrCHJqB VrWsgMC\%2F4dy9qJJFTkna2

[15] BT Wholesale Radio Backhaul Service (RBS), Backhaul Billing Examples.

http://www.btwholesale.com/pages/static/Pricing and Contracts/Refer ence Offers/Radio Base Station Backhaul Services.html 